\title{
Intelligent Computing Methods for The Interpretation of Neuropsychiatric Diseases Based on RBR-CBR-ANN Integration
}

\author{
Mohit Gangwar, R. B. Mishra, R. S. Yadav, B. Pandey \\ Department of CSE, MNNIT, Allahabad, India \\ mohitgangwar@gmail.com \\ Department of Computer Engineering, IIT-BHU, Varanasi, India \\ head.cse@itbhu.ac.in \\ Department of CSE, MNNIT, Allahabad, India \\ yadavrs64@gmail.com
}

\begin{abstract}
Neuropsychiatry is an integrative and collaborative field that brings together brain and behavior, but its diagnosis is complex and controversial due to the conflicting, overlapping and confusing nature of the multitude of symptoms, hence the need to retain cases in a case base and reuse effective previous solutions for current cases. This paper proposes a method based on the integration of Rule based reasoning (RBR), Case based reasoning (CBR) and Artificial neural network (ANN) that utilizes solutions to previous cases in assisting neuropsychiatrist in the diagnosis of neuropsychiatric diseases. The system represents five neuropsychiatric diseases with 38 symptoms grouped into six categories. Integrated method improves the computational and reasoning efficiency of the problem-solving strategy. The basic objective of this work is to develop integrated model of RBR-CBR and RBR-CBR-ANN in which RBR is used to hierarchically correlate the sign and symptom of the disease and also to compute CCF of the diseases. CBR is used for diagnosing the neuropsychiatric diseases for absolute and relative diagnosis. In relative diagnosis CBR is also used to find the relative importance of sign and symptoms of a disease to other disease. ANN and heuristic method used in matching and selection process in CBR.
\end{abstract}

\section{Keywords}

neuropsychiatric diseases; Case-based reasoning; Rule-based reasoning; Artificial neural network; Absolute diagnosis; Relative diagnosis; EEG; neuroimagin; FMRI; cognitive; psychological; Physiological.

\section{Council for Innovative Research}

Peer Review Research Publishing System

Journal: INTERNATIONAL JOURNAL OF COMPUTERS \& TECHNOLOGY

Vol 11, No. 5

editor@cirworld.com

www.cirworld.com, member.cirworld.com 


\section{INTRODUCTION}

Intelligent computing methods for the solution of complex problem of heuristic nature are: KBS (rule-based), casebased reasoning (CBR), modal-based reasoning (MBR), artificial neural network (ANN) and genetic algorithm (GA). The first three are basically grouped under knowledge enriched methodology, where as ANN and GA are grouped under data dominant methodology. The integration of a computing method from any one of the methods from knowledge group to any method of data dominant group improves efficiency and accuracy of the result at the cost of increased computational complexity and programmability [1]. The integration of knowledge enriched and data dominant computing methods are being extensively used now days for the detection, diagnosis and treatment of many diseases in neurology and psychiatry etc.

The integration of rule-based and ANN method has been deployed for robotic control [2] and prediction of hepatitis infection [3]. Rule-based reasoning (RBR) and case-based reasoning (CBR) have been used for diagnosis of Alzheimer's disease patients [4] and automated sleep stage scoring [5]. But integration of RBR, CBR and ANN has been rarely used in neuropsychiatry.

Neuropsychiatry is an integrative and collaborative field that brings together brain and behavior. Yudofsky and Hales [6] described the underlying tenets of the discipline to be the "inseparability of brain and thought, of mind and body, and of mental and physical". Neuropsychiatry attempts to bridge the artificial boundaries between neurology and psychiatry in order to treat the multitude of clinical manifestations of the singular brain. Neuropsychiatry is primarily focused on the assessment and treatment of the cognitive, behavioral, and mood symptoms of patients with neurological disorders. However, an equally important focus for neuropsychiatrists is the understanding of the role of brain dysfunction in the pathogenesis of primary psychiatric disorders [7].Therefore, not only does neuropsychiatry bring the psychiatric assessment and treatment of psychotic or mood symptoms to the neurology arena, it also does neuropsychiatry bring the psychiatric assessment and treatment of psychotic or mood symptoms to the neurology arena, it also returns clinicians to the objective rigors of physical diagnosis and testing, which are often not practiced in psychiatry today [8].

Neuropsychiatric diagnosis is based on elicitation of clinical symptoms, identification of neuropsychiatric syndromes, construction of a differential diagnosis, use of laboratory tests and neuroimaging to support or exclude specific diagnoses, and identification of the primary etiology of the behavioral disturbance. In some cases, longitudinal assessment and careful monitoring of treatment responses may be necessary to clarify obscure diagnosis. Treatment depends on accurate diagnosis [9].

The neuropsychiatric examination is a specialized clinical evaluation that supports clinical diagnosis and management in behavioral disease [10]. The elements of the neuropsychiatric examination include the psychiatric evaluation described in the American Psychiatric Association Practice guidelines for the psychiatric evaluation; the neurologic examination [11]; and the neurobehavioral mental status examination [12] [13] [14]. This examination includes neuroimaging and neurophysiologic testing.

The neuropsychiatric examination differs from conventional psychiatric evaluation by its inclusion of cognitive assessment, the neurological examination, and attention to supportive neuroimaging and/or neurophysiologic test data. In addition, the neuropsychiatric examination aims to produce and explicit neurobehavioral formulation of behavioral disorders, which identifies neuroanatomy, neurophysiology, and neurochemistry of relevance to the behavioral syndrome at issue. The management plans that evolve from neuropsychiatric examinations typically include a border range of therapeutic options than those of general psychiatry, often including the range of available neurotropic drugs in addition to the psychotropic agents [15].

Sign and symptoms plays very important role in the detection and diagnosis of neuropsychiatric diseases. Apart from EEG and neuroimagin parameters and features, physiological, psychological and cognitive parameters are also of prime importance. The important parameters of EEG for detection and diagnosis of neuropsychiatric abnormality are: frontal, parietal, occipital and temporal [16]. Neuroimagin parameters are: frontal, anterior cingulate cortex, cingulate gyms, parietal, occipital, temporal and basal ganglion [17]. Physiological parameters are: Muscle weakness and movement difficulty; psychological parameters are concerned with Hallucination, Hyper activity, Anxiety, Frustration and Delusion etc [18] [19] [20]; cognitive parameters depict of Speech disability, Reasoning and Learning disability etc [18] [19]. The combination of EEG parameters, neuroimagin parameters, physiological, psychological and cognitive parameters are required for the detection of neuropsychiatric diseases such as: Attention deficit hyperactivity disorder, Dementia, Mood Disorder, Obsessive-compulsive disorder and Schizophrenia.

The rule-based/knowledge-based models in general used for diagnosing and interpretation. Meddahi et al. developed approach for knowledge acquisition using multi-channel electroencephalogram interpretation [21].

ANN methodologies have also been deployed for detection of neurological, psychiatric and neuropsychiatric diseases. García-Pérez et al. [22] used neural networks for differential diagnosis of Alzheimer disease and vascular dementia. Zou et al. [23] classified psychiatric diseases using artificial neural network. Li and Fan [24] described a model for classification of Schizophrenia and depression by EEG with ANNs. Yana et al. [25] used a three-layered perceptron and interview based neural network classifier for different eatiating psychotic disorders (Mood Disorder and Schizophrenia) for neurotic 
disorders. Vollala and Gulla [26] used neural network approach for automatic detection of EPILEPSY form EEG signal which uses approximate entropy as the input feature. Lowell and Davis [27] used feed-forward neural network for classifying the group of psychiatric diseases.

ANN-CBR combined method is used for heart transplant [28]. This method described learning general knowledge in a diagnostic case-based system through the use of a neural network. Haraldsson et al. [29] present a case-based method that qualitatively explains the operation of the ANNs, by showing regions of each ECG critical for ANN response.

Hardly, there is a method which deals with the combination of ANN to RBR or ANN to CBR for the diagnosis of neuropsychiatric diseases.

In this work we have developed integrated model consisting of rule-based and CBR for absolute diagnosis and integrate mode consisting of RBR, CBR and ANN for relative diagnosis. Diagnosis and interpretation of different neuropsychiatric diseases based upon EEG, neuroimagin; psychological, cognitive and physiological parameters. The objective of our work as follow:

- $\quad$ To develop integrated of RBR and CBR in which RBR is used to hierarchically correlate the sign and symptom of the disease and also to compute cumulative confidence factor (CCF) of the disease for absolute diagnosis. CBR is used for the generation of case base and diagnosing neuropsychiatric disease.

- $\quad$ To develop integrated model of RBR, CBR and ANN in which RBR is used to hierarchically correlate the sign and symptom of the disease and also to compute cumulative confidence factor (CCF) of all the diseases for differential diagnosis. CBR is used for diagnosing the neuropsychiatric diseases and to find the relative importance of sign and symptoms of diseases to other disease and ANN is used for matching process in CBR.

- In differential diagnosis to determine the highest CCF which corresponds to sign and symptoms depicted in EEG signal, and parameters of neuroimagin, physiological, psychological and cognitive origin of a particular patient given by user

- $\quad$ To show the effectiveness of combining rule-based model, CBR and incorporation of ANN in CBR in the diagnosis process of neuropsychiatric diseases.

The organization of the paper as follows. Section 2 presents the description of the various neuropsychiatric diseases with their physiological, psychological, and cognitive parameters. The EEG and neuroimagin parameters are also mentioned in this context. Section 3 describes the rule-based model for correlating the diseases. Section 4 deals with the CBR model. Section 5 and 6 shows the computation and results for absolute as well as relative diagnosis respectively. Section 7 deals with conclusion.

locomotion

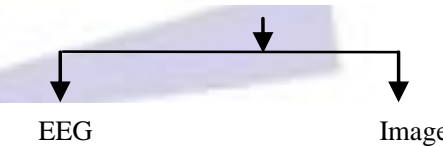

Characteristics Characteristics
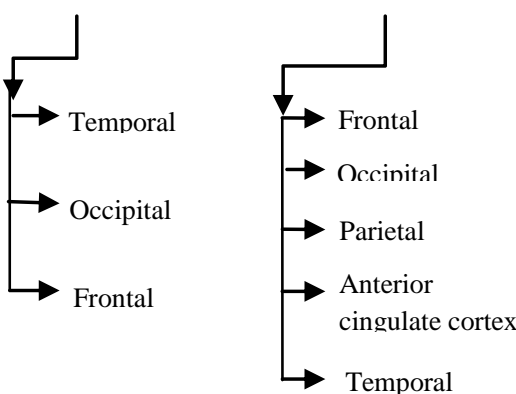

Social withdrawal

Fig 1: Hierarchical correlation of sign and symptoms (evidence) of the Schizophrenia disease 


\section{PROBLEM DESCRIPTION}

Problem description table (Table 1) describes diseases with their two important parameters: physio-psycho (PP) parameters, signal characteristics. Physio-psycho parameter is further divided into three parts: (i) muscular physiology (M Phy) consisting of muscular (M) parameters such as: oversleeping (OS) and muscle weakness (MW); and motor activity (MA) parameters such as: difficulty in movement (MO), difficulty in locomotion (LO), difficulty in using toilet (UT), difficulty in climbing stairs (CS), difficulty in walking (W); (ii) cognitive parameters are confusion in decision making (CD), hearing disability (HD), forgetting memory (FM), judgment (JG), learning disability (LD), reasoning (RS), speech disability (SD) and vision disability (VD); (iii) psychological parameters are: distraction of work (DW), hallucination (HL), fear (FR), hyper activity (HA), agitation $(A G)$, anxiety (AX), stress (ST), anger (AN), abnormal behavior (AB), need of perfection (NP), social withdrawal (SW) and delusion (DE). The signal characteristic is also divided into two parts; (i) EEG signal abnormality observed in following brain regions such as: frontal $(\mathrm{FL})$, parietal $(\mathrm{PL})$, occipital $(\mathrm{OL})$ and temporal $(\mathrm{TL})$; and (ii) neuroimagin detect abnormality in these brain regions such as: frontal $(F L)$, anterior cingulate cortex $(A C)$, cingulate gyms $(C G)$, parietal $(P L)$, occipital $(O L)$, temporal (TL) and basal ganglion (BG). In Table 1, row represents the disease and the column represents their respective parameters. The sub columns of muscular, motor action, cognitive and psychological parameter contains " $Y$ " if the symptom is present in the disease shown in respective row. For example, Schizophrenia has muscular symptoms such as: over sleep. Therefore these columns contain " $Y$ " as in Table 1. Similarly, the forgotten memory and speech disability sub-column of cognitive contains " $Y$ ". Finally the table is formed with five rows corresponding to five diseases and 38 columns corresponding to the parameters of the diseases.

We have organized evidences (symptoms) of Schizophrenia disease in hierarchical tree (Figure 1) which is composed of basic evidences (symptoms) and nodes. Each node corresponds to a rule in the rule-base. The if (condition) of a rule relates parameters by mean of the logical operators (and). These parameters are the branches that arrive to a node, that is, the evidences and the result of previous nodes. The system works with certainty factor (CF) which are equivalent to values associated to nodes and evidences represented in the hierarchical tree. When a condition is satisfied, a resulting CCF is obtained from the CF of each entry parameter. The value attained to a node represents the maximum CCF that may be achieve by this node, that is, when all parameters are satisfied in a particular disease. The final result is obtained by the execution of all the pertinent rules.

Table 1. Problem description table (PDT)

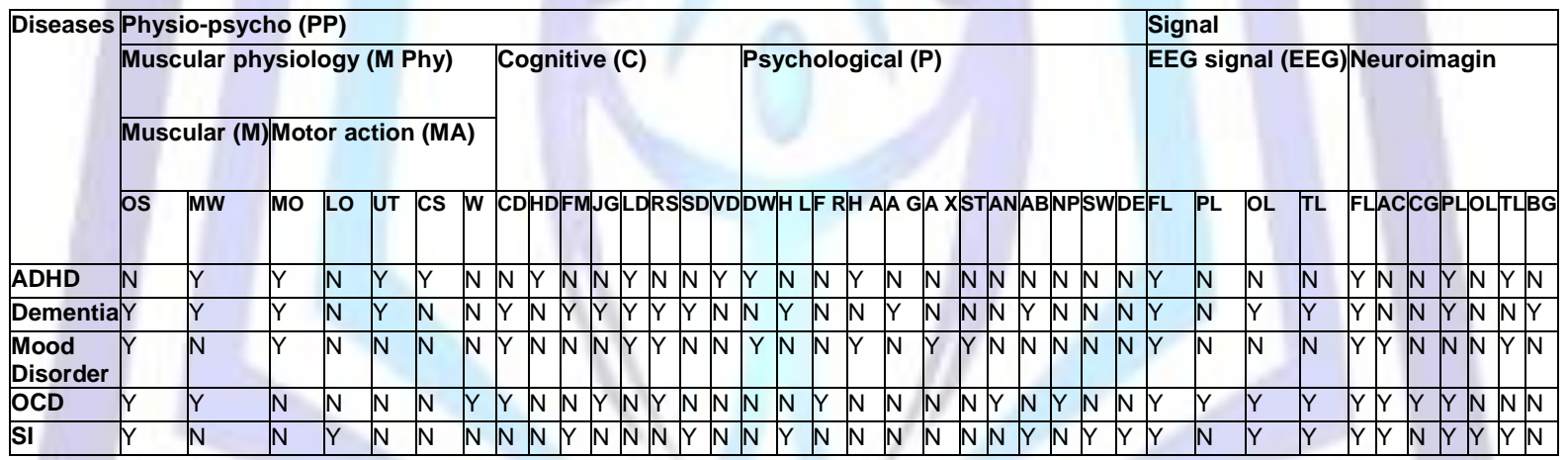

\section{RULE-BASED MODEL}

The rule-based model is developed for Schizophrenia disease on the basis of the hierarchal tree showing the parameters and their correlation in a particular disease. Figure 2 shows the rules generated form hierarchical tree for Schizophrenia. Similarly we can generate rules for other diseases. Table 2 shows the hierarchal rules generated based on hierarchal tree structure for all diseases. It is clear from Table 2 that, when rules $R_{1111}$ and $R_{1112}$ are satisfied then $R_{111}$ is satisfied; when $R_{111}, R_{112}$ and $R_{113}$ are satisfied then $R_{11}$ is satisfied; when $R_{121}$ and $R_{122}$ are satisfied then $R_{12}$ is satisfied and when $R_{11}$ and $R_{12}$ are satisfied then $R_{1}$ is satisfied.

$$
\begin{aligned}
& \mathrm{R}_{1111} \oplus \mathrm{R}_{1112} \rightarrow \mathrm{R}_{111} \\
& \mathrm{R}_{111} \oplus \mathrm{R}_{112} \oplus \mathrm{R}_{113} \rightarrow \mathrm{R}_{11} \\
& \mathrm{R}_{121} \oplus \mathrm{R}_{122} \rightarrow \mathrm{R}_{12} \\
& \mathrm{R}_{11} \oplus \mathrm{R}_{12} \mathrm{R}_{1}
\end{aligned}
$$

Similarly, others rules of Table 2 are generated to any lower level to upper level. In Figure 2 the CF shown in brackets is used for computing CCF. Rule-based model shows modular representation of the facts. Modules can be hierarchically related as shown in tree structure. Sign and symptoms at the lowest level are correlated to the parameters at the higher level and also the lower level parameters to the higher level parameters. In this way, going from bottom to up i.e. lower to 
higher level and after travel of certain levels we reach to the highest level i.e. root level, which concerns with a type of disease with a CCF. The calculation of CF in cumulative fashion is termed as CCF.

The CCF value of each level in a hierarchical tree is computed using formula [30] as follows:

$M B(h, e 1 \&$ e2 $)=M B(h, e 1)+M B(h, e 2)(1-M B(h, e 2))$

Where in a rule-base model e1 may represent a set of rules whose cumulative effects have previously been considered and e2 represents new rule whose effect is to be added to the previously existing belief. Similarly,

$M D(h, e 1 \&$ e2) $=M D(h, e 1)+M D(h, e 2)(1-M D(h, e 2))$

$M D(h, e 1 \&$ e2) $=0$, If $M B(h, e 1 \&$ e2) $=1$ i.e. all the evidences (e1 \& e2) approves the hypothesis $(h)$.

$C C F=M B(h, e 1 \&$ e2 $)+M D(h$, e1 \& e2)

Rule 1: IF over sleep (z1) THEN muscular abnormality $(z)$
Rule 2: IF there is difficulty in locomotion ( $z 1)$ THEN motor action abnormality (z)

Rule 3: IF there is muscular abnormality (z1) \& IF motor action abnormality (z2) THEN muscular physiology abnormality $(z)$

Rule 4: IF Speech disability (z1) \& IF forgetting memory (z2) THEN cognitive abnormality (z)

Rule 5: IF Hallucination (z1) \& IF abnormal behaviour (z2) \& IF social withdrawal (z3) \& IF delusion (z4) THEN psychological abnormality (z)

Rule 6: IF there is muscular physiology abnormality (z1) \& IF cognitive abnormality (z2) \& IF psychological abnormality (z3) THEN physio-psycho abnormality (z)

Rule 7: IF Frontal (z1) \& IF occipital (z2) \& IF temporal (z3) THEN EEG abnormality (z)

Rule 8: IF Frontal (z1) \& IF anterior cingulate cortex (z2) \& IF parietal (z3) \& IF occipital (z4) \& IF temporal (z5) THEN Image abnormality $(z)$

Rule 9: IF EEG abnormality (z1) \& IF image abnormality (z2) THEN signal abnormality (z)

Rule 10: IF Physio-psyco abnormality (z1) \& IF there is signal abnormality (z2) THEN disease is Schizophrenia (z)

Fig 2: Rules for diagnosing Schizophrenia disease

\section{CBR MODEL}

$\mathrm{CBR}$ is a tabular form of representation of cases (diseases) with their parameters. The selection and matching of a particular unknown disease on the basis of sign and symptoms with the content of a case-base is performed in CBR module. In CBR model the first process is to representation of knowledge and acquisition. Knowledge representation and acquisition process is described in this section. Remaining CBR process i.e. case-base generation, matching and adaptation implemented in computation process for absolute as well as relative diagnosis.

\subsection{Knowledge Representation and Acquisition}

The knowledge is represented via feature-vector and stored in a database in tabular form. The knowledge is acquired through dialogue session as shown below. 
Table 2. Hierarchal rules structure (Rule base)

ADHD

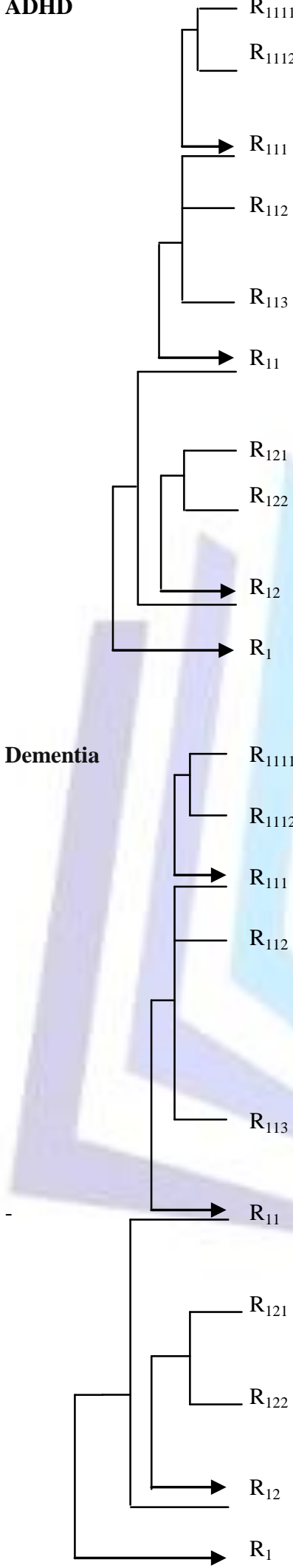

\section{IF}

Muscle weakness (z1)

Difficulty in movement (z1)

Difficulty in using toilet (z2)

Difficulty in climbing stairs (z3)

Muscular abnormality (z1)

Motor action abnormality (z2)

Hearing disability (z1)

Learning disability (z2)

Visual disability (z3)

Distract from work (z1)

Hyperactive (z2)

Muscular physiological abnormality (z1)

Cognitive abnormality (z2)

Psychological abnormality (z3)

Frontal observed (z1)

Frontal observed (z1)

Parietal observed (z2)

Temporal observed (z3)

EEG abnormality (z2)

Image abnormality (z3)

Physio-psycho abnormality (z1)

Signal abnormality (z2)

Over sleep (z1)

Muscular weakness (z2)

Difficulty in movement (z1)

Difficulty in using toilet (z2)

Muscular abnormality (z1)

Motor action abnormality (z2)

Decision making inability (z1)

Judgment inability (z2)

Learning Disability (z3)

Forgetting memory (z4)

Reasoning (z5)

Speech disability (z6)

Hallucination (z1)

Abnormal behaviour (z2)

Agitation (z3)

Muscular physiological abnormality (z1)

Cognitive abnormality (z2)

Psychological abnormality (z3)

Frontal observed (z1)

Occipital observed (z2)

Temporal observed (z3)

Frontal observed (z1)

Parietal observed (z2)

Basal ganglion observed (z3)

EEG abnormality (z1)

Image abnormality (z2)

Physio-psycho abnormality (z1)

Signal abnormality (z2)

\section{THEN}

Muscular abnormality (z)

Motor action abnormality (z)

Muscular physiological abnormality (z)

Cognitive abnormality (z)

Psychological abnormality (z)

Physio-psycho abnormality (z)

EEG abnormality (z)

Image abnormality (z)

Signal abnormality (z2)

Attention deficit hyperactivity disorder (z)

Muscular abnormality (z)

Motor action abnormality (z)

Muscular physiological abnormality (z)

Cognitive abnormality (z)

Psychological abnormality (z)

Physio-psycho abnormality (z)

EEG abnormality (z)

Image abnormality $(\mathrm{z})$

Signal abnormality $(\mathrm{z})$

Dementia (z) 
Table 2. (continued)

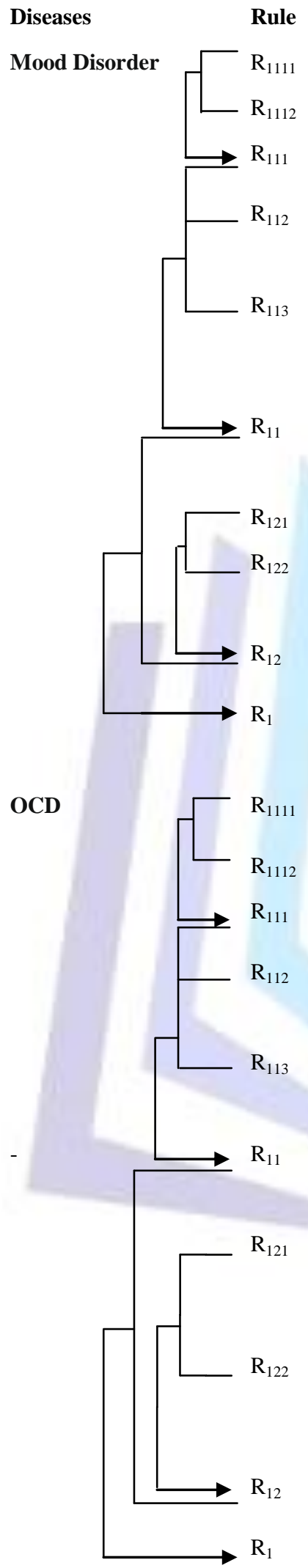

\section{IF}

Over sleep (z1)

Difficulty in movement (z1)

Muscular abnormality (z1)

Motor action abnormality (z2)

Decision making inability (z1)

Learning disability (z2)

Reasoning (z4)

Distraction of work (z1)

Hyper activity (z2)

Anxiety (z3)

Stress (z4)

Muscular physiological abnormality (z1)

Cognitive abnormality (z2)

Psychological abnormality (z3)

Frontal observed (z1)

Frontal observed (z1)

Anterior cingulate cortex observed (z2)

Temporal observed (z3)

EEG abnormality (z1)

Image abnormality (z2)

Physio-psycho abnormality (z1)

Signal abnormality (z2)

Over sleep (z1)

Muscular weakness (z2)

Difficulty in walking (z1)

Muscular abnormality (z1)

Motor action abnormality (z2)

Decision making inability (z1)

Judgment inability (z2)

Reasoning (z3)

Frustration (z1)

Anger (z2)

Need of perfection $(\mathrm{z} 3)$

Muscular physiological abnormality (z1)

Cognitive abnormality (z2)

Psychological abnormality (z3)

Frontal observed (z1)

Parietal observed (z2)

Occipital observed (z3)

Temporal observed (z4)

Frontal observed (z1)

Anterior cingulate cortex observed (z2)

Cingulate gyms observed (z3)

Parietal observed (z4)

EEG abnormality (z1)

Image abnormality (z2)

Physio-psycho abnormality (z1)

Signal abnormality (z2)

\section{THEN}

Muscular abnormality (z)

Motor action abnormality (z)

Muscular physiological abnormality (z)

Cognitive abnormality (z)

Psychological abnormality (z)

Physio-psycho abnormality (z)

EEG abnormality (z)

Image abnormality (z)

Signal abnormality (z)

Mood Disorder (z)

Muscular abnormality (z)

Motor action abnormality (z)

Muscular physiological abnormality (z)

Cognitive abnormality (z)

Psychological abnormality (z)

Physio-psycho abnormality (z)

EEG abnormality (z)

Image abnormality (z)

Signal abnormality (z)

Obsessive-compulsive disorder (z) 
Table 2. (continued)

Schizophrenia

\section{THEN}

Muscular abnormality (z)

Motor action abnormality (z)

Muscular physiological abnormality (z)

Cognitive abnormality (z)

Psychological abnormality (z)

Physio-psycho abnormality (z)

EEG abnormality (z)

Image abnormality (z)

Signal abnormality (z)

Schizophrenia (z)

\subsubsection{Physio-Psycho parameter}

Physio-psycho parameter consists of muscular physiology parameter i.e. muscular and motor action parameter, cognitive and psychological parameter.

\section{Muscular features}

Is the patient suffering with oversleep problem? Is the patient suffering with muscle weakness?

$\mathrm{Y}:[\mathrm{OS}=1]$

$\mathrm{Y}:[\mathrm{MW}=1]$

\section{Motor action parameters}

Is the patient having difficulty in movement? Is the patient having difficulty in locomotion? Is the patient having difficulty in using toilet? Is the patient having difficulty in climbing stairs? Is the patient having difficulty in walking?

$\mathrm{Y}:[\mathrm{MO}=1]$

$Y:[L O=1]$

$Y:[U T=1]$

$Y:[C S=1]$

$\mathrm{Y}:[\mathrm{W}=1]$

\section{Cognitive parameter}

Does the patient have visual disability?

Does the patient have speech disability?

Does the patient have hearing disability?

Does the patient have learning disability?

Does the patient have confusion in decision making?

Does the patient have confusion in Judgment?

Does the patient have problem of forgetting memory?

Does the patient have problem in reasoning?
$\mathrm{Y}:[\mathrm{VD}=1]$
$Y:[S D=1]$
$Y:[H D=1]$
$Y:[L D=1]$
$Y:[C D=1]$
$Y:[J G=1]$
$Y:[F M=1]$
$Y:[R S=1]$ 


\section{Psychological parameter}

Does the patient have feeling of anger?

Does the patient have feeling of anxiety?

Does the patient have feeling of agitation?

$\mathrm{Y}:[\mathrm{AN}=1]$

Does the patient suffering from delusion?

$Y:[A X=1]$

$Y:[A G=1]$

$Y:[D E=1]$

Does the patient suffering from hallucination? $\quad \mathrm{Y}:[\mathrm{HL}=1]$

Does the patient have abnormal behaviour? $\quad \mathrm{Y}:[\mathrm{AB}=1]$

Does the patient feel fear?

$\mathrm{Y}:[\mathrm{FR}=1]$

Does the patient distract from work?

Does the patient is hyperactive?

Does the patient feel social withdrawal?

$Y:[D W=1]$

$Y:[H A=1]$

$Y:[S W=1]$

Does the patient needs of perfection?

$Y:[N P=1]$

Does the patient feels stressed?

$Y:[S T=1]$

\subsubsection{Signal parameter}

Signal parameter consists of mainly two parts: EEG signal characteristics and neuroimagin parameters.

\section{EEG related features}

Is Frontal observed in EEG?

Is Parietal observed in EEG?

Is Occipital observed in EEG?

Is Temporal observed in EEG?

$Y:[F L=1]$

$Y:[P L=1]$

$Y:[\mathrm{OL}=1]$

$\mathrm{Y}:[T L=1]$

\section{Neuroimagin related parameters}

Is anterior cingulate cortex observed in image?

$Y:[A C=1]$

$Y:[C G=1]$

$Y:[F L=1]$

$\mathrm{Y}:[\mathrm{PL}=1]$

$Y:[\mathrm{OL}=1]$

$Y:[T L=1]$

$Y:[B G=1]$

Is Frontal observed in image?

tetal observed in image?

Is Occipital observed in image?

Is basal ganglion observed in image?

These symptoms take the value "one" or "zero" for the presence and absence of the symptoms, respectively.

\section{COMPUTATION FOR ABSOLUTE DIAGNOSIS}

Computation is implemented using Java Language. The computation is done in the following steps: Computation of input (X) matrix, generation of patient data vector (PDV), retrieval, adaptation and computation of CCF of the diagnosed disease. Figure 4 shows the entire step in order and Figure 5 shows the flowchart for the implementation of the system.

\subsection{Case Storage}

Cases are stored in the tabular form i.e. computation of input matrix $X$ and output. Following steps are used to compute any element $x_{i j}$ of $\mathrm{X}$ matrix:

All the main six parameters as given in Table 1 i.e. muscular, motor action activity, psychological, cognitive, EEG characteristics and neuroimagin characteristics are represented by string of binary numbers where the number of bits ( $n$ ) in the string represents the number of sub-parameters (symptoms) considered under the particular parameter. For example, muscular parameter consists of oversleeping and muscle weakness therefore a binary string of 2 bits is used to represent muscular parameter.

1. The binary string contains 1 and 0 for " $Y$ " and " $N$ " respectively as in Table 1.

2. The binary string is then converted in decimal value.

3. The decimal value is then divided by $2^{n}$.

The data of five cases are shown in Table 2. In Table 2, column headed by F1, F2, F3, F4, F5 and F6 contains the values obtained by applying the step 1 to 3 as described above for muscular, motor, cognitive, psychological, EEG and neuroimagin parameters respectively.

For example, in fifth case: i.e. Schizophrenia.

The value of $\mathrm{F} 1$ :

The sub column of muscular parameter in Schizophrenia i.e. over sleeping and muscle weakness contains $\mathrm{YN}$ as shown in Table 1. The steps from 1 to 3 given above are used to calculate F1. 
- Replace $\mathrm{Y}$ by 1 and $\mathrm{N}$ by 0 then the string is " 10 ".

- The decimal value of " 10 "is 2 .

- $\quad$ Divide the decimal value i.e. 2 by $2^{n}\left(\right.$ i.e. $2^{2}=4$ ) we get 0.5

Similarly, we can compute all the values for Table 3.

Table 3. Case-base for absolute diagnosis

\begin{tabular}{|l|l|l|l|l|l|l|l|}
\hline Cases & F1 & F2 & F3 & F4 & F5 & F6 & Output \\
\hline Case1 & 0.25 & 0.688 & 0.2851 & 0.563 & 0.5 & 0.578 & ADHD \\
\hline Case2 & 0.75 & 0.625 & 0.742 & 0.283 & 0.6875 & 0.570 & Dementia \\
\hline Case3 & 0.5 & 0.5 & 0.547 & 0.586 & 0.5 & 0.766 & MD \\
\hline Case4 & 0.75 & 0.031 & 0.578 & 0.129 & 0.9375 & 0.937 & OCD \\
\hline Case5 & 0.5 & 0.25 & 0.1328 & 0.253 & 0.6875 & 0.859 & SI \\
\hline
\end{tabular}

\subsection{Generate PDV (New case)}

PDV (new case) is generated from user input. The pseudo code for generating PDV is shown in Figure 3 on the basis of programming steps as shown below.

1. PDV consists of six parameters: muscular, motor action activity, cognitive, psychological, EEG characteristics and Image characteristics.

2. Represent all the parameters by sub-string of binary numbers $\left[s_{1}(n=2), s_{2}(n=5), s_{3}(n=8), s_{4}(n=12), s_{5}(n=4)\right.$, $\left.s_{6}(n=7)\right]$ where $s_{1}, s_{2}, s_{3}, s_{4}, s_{5}$ and $s_{6}$ are sub string for muscular, motor action activity, cognitive, psychological, EEG characteristics and image characteristics respectively, and $n$ is number of bits (sub-parameters/symptoms) in a sub string.

3. The bits in sub string are set to 1 or 0 indicating the presence (selection of the condition) and absence of the symptom.

4. The binary sub-strings are then converted into decimal value.

5. The decimal values are then divided by $2^{n}$, denote the result by $[n 1, \ldots, n 6]$.

6. The result $[n 1, n 2, n 3, n 4, n 5, n 6]$ is a PDV.

A new PDV $\{0.5,0.25,0.1328125,0.25268555,0.6875,0.859375\}$ i.e. new case is generated (as shown at the bottom most position of the left side of the Figure 4) corresponding to sign and symptoms selected in the upper part of left side in Figure 4.

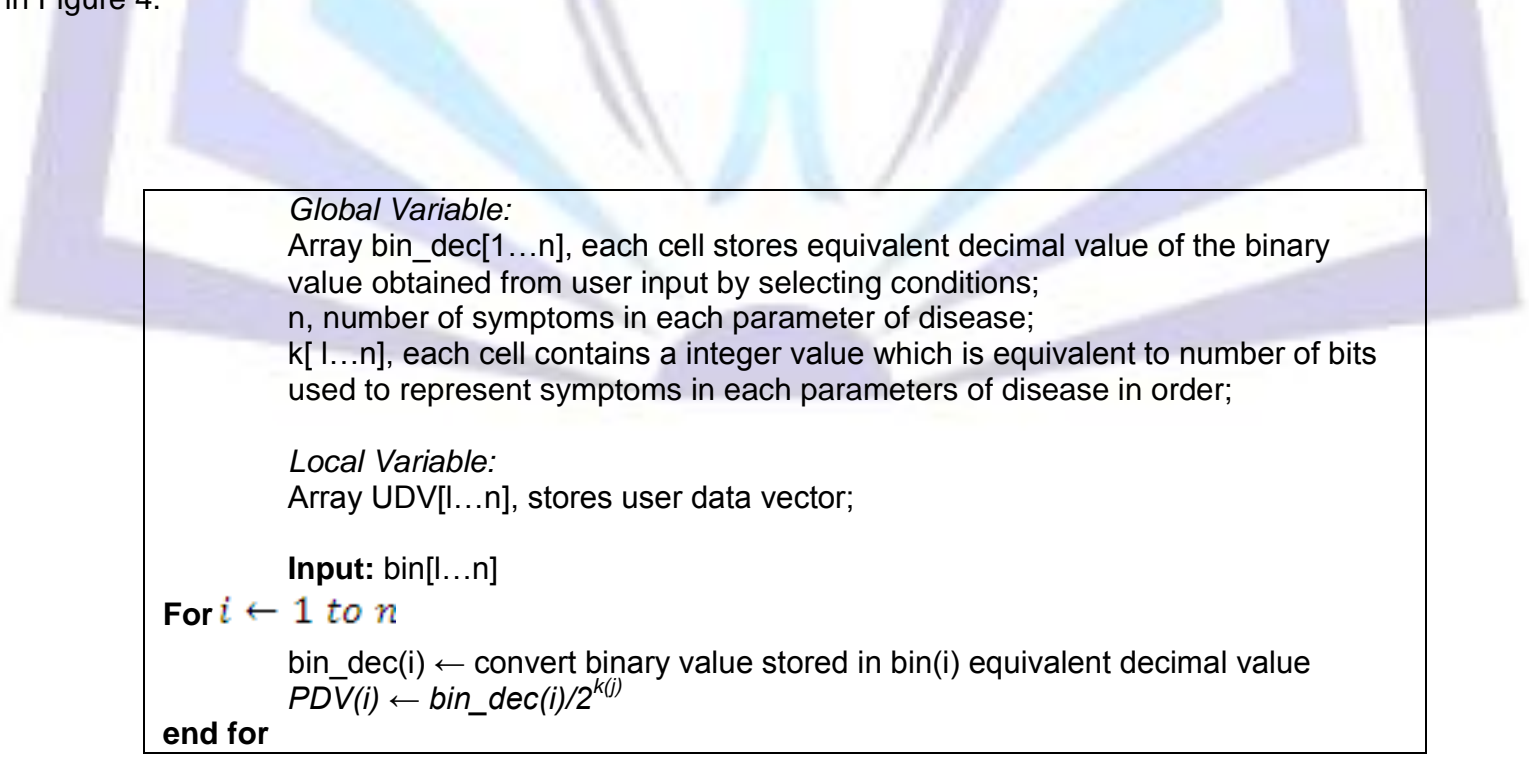

Fig 3: Pseudo code for generating PDV 


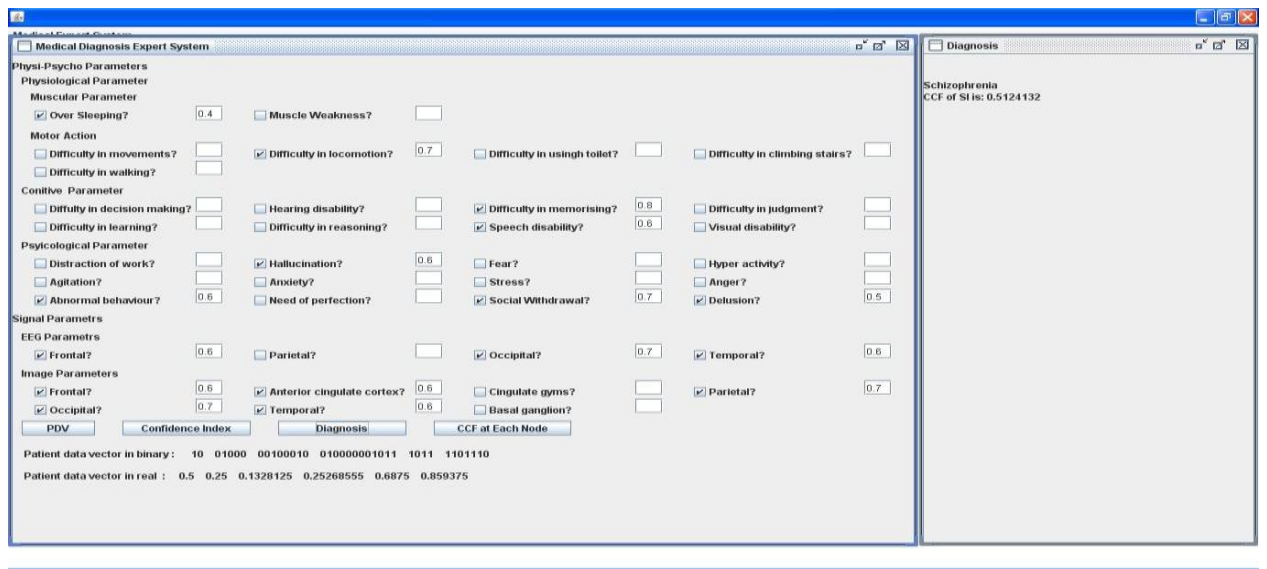

Fig 4: Computation result for absolute diagnosis

\subsection{Case Retrieval}

The retrieval consists of two steps: matching and selection.

1. The distance between $\mathrm{i}^{\text {th }}$ features of two cases is computed based on the following equations:

$$
D V_{i}=\left|C B_{i}-N C_{i}\right| ; \quad(i=I, \ldots \ldots ., n)
$$

With $n$ : the entire feature count. $D V_{i}$ : the $i^{\text {th }}$ distance value between two $i^{\text {th }}$ symptoms. $C B_{i}$ : the $i^{\text {th }}$ value of feature of existing case (patient) in the case-base. $\mathrm{NC}_{\mathrm{i}}$ : the $\mathrm{i}^{\text {th }}$ value of feature of new case (patient).

2. The similarity measure between two cases. Let SVj be the similarity value between the new inputted case and the $\mathrm{j}^{\text {th }}$ case in the case-base. $\mathrm{SV}_{\mathrm{j}}$ can be calculated as follows:

$$
\mathrm{SW}=\sum_{j=1}^{\mathrm{n}} D V_{j}
$$

With $\mathrm{j}=\mathrm{l}, \ldots, \mathrm{k}$, where $\mathrm{k}$ is the number of cases in the case-base.

The new PDV is matched with all the old cases in case-base as input matrix (X) using the equation (4) and (5) as shown in the Table 3. This PDV is most similar to the fifth case of Table 3.

\subsection{Case Adaptation}

The purpose of the case adaptation is to reuse the retrieved case without any modification to solve the new problem. Null adaptation is applied for this purpose.

\subsection{Result and Cumulative Confidence Factor Computation}

The CF shown in brackets, in Figure 2 is used for computing CCF. Rule-based model shows modular representation of the facts. Modules can be hierarchically related as tree structure shown in Figure 1 and Table 2. Sign and symptoms at the lowest level are correlated to the parameters at the higher level and also the lower level parameters to the higher level parameters. In this way, going from bottom to up i.e. lower to higher level and after travel of certain levels we reach to the highest level i.e. root level, which concerns with a type of disease with a CCF. The calculation of CF in cumulative fashion is termed as CCF.

After selecting row in case-base which is similar to the new PDV1, RBR applied to compute the CCF of selected disease on the basis of selected symptoms used to generate PDV1 by applying the formulas given in Section 3 on the hierarchical tree.

In rule -based model different weight are assigned to different parameters at different node (rule) depending upon how much the particular parameters contributes in the diagnosis of disease such as: the physio-psycho parameters play more important role then signal parameters in diagnosis the diseases therefore, the weight assigned to physio-psycho and signal parameters are $2 / 3$ and $1 / 3$, respectively. Among three sub parameters: muscular physiology, cognitive and psychological of physio-psycho parameter, the first parameter has more importance than latter two. Therefore the weight assigned to muscular physiology, cognitive and psychological are 2/3,1/6 and 1/6 respectively. Similarly the muscular and motor action parameters contributes equally in muscular physiology therefore, the weight assigned to both are $1 / 2$. 
Similarly, EEG characteristic contributes less than neuroimagin characteristics in signal parameters therefore the weight assigned to them are $1 / 3$ and $2 / 3$, respectively. These weights are used in the computation of CCF.

CCF is computed from bottom to top in hierarchal tree. The CF of leaf nodes are given as shown in Figure 6 . The CCF at various nodes i.e. muscular, motor action activity, cognitive, psychological, EEG parameters and neuroimagin parameters are computed by using equations (1), (2) and (3).

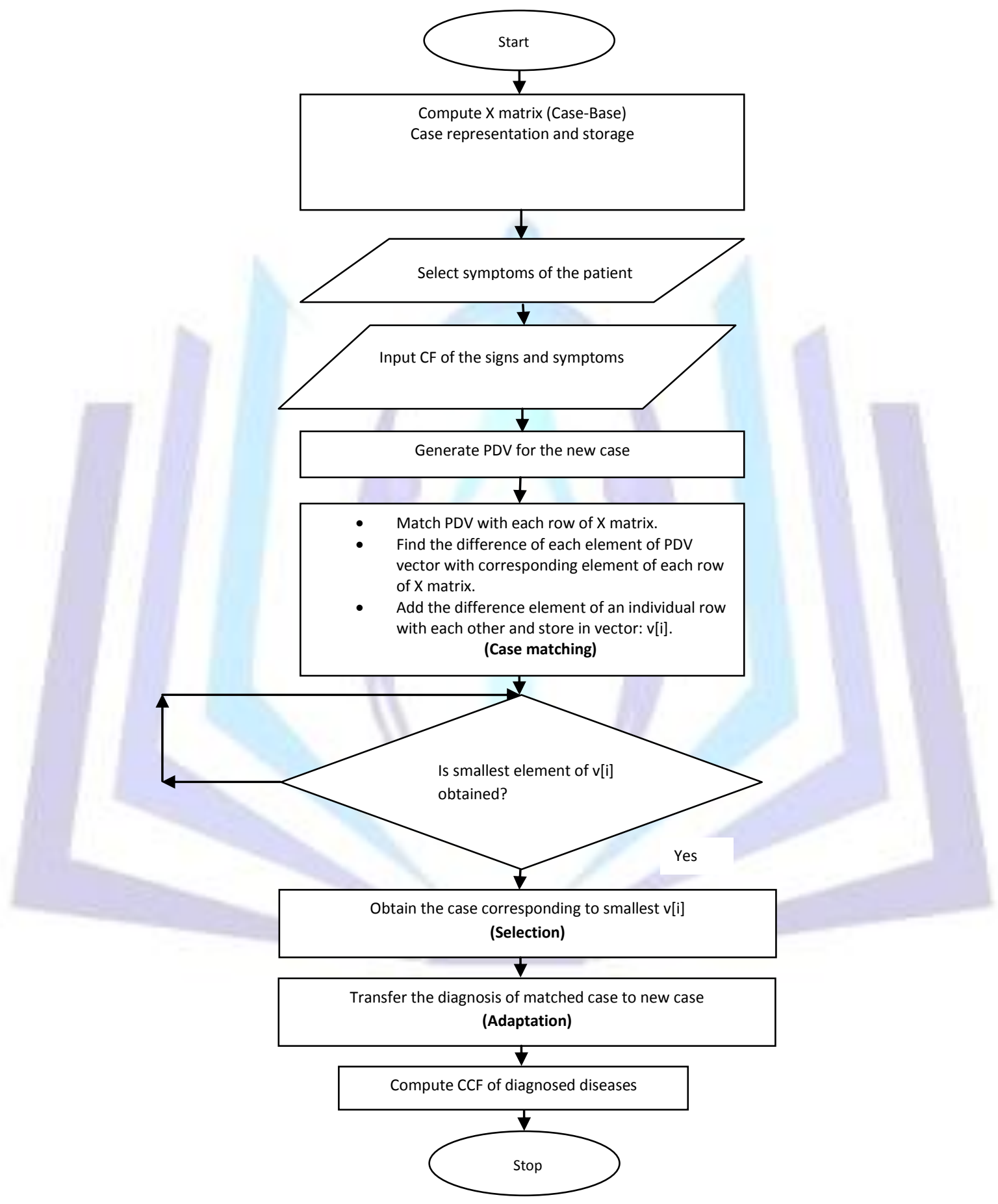

Fig 5: Flowchart for the implementation of absolute diagnosis 
For example, in hierarchal tree of Schizophrenia the evidence (e) in support of rule $R_{1111}$ are e1: over sleep $(z 1=0.4)$, e2: muscular weakness $(\mathrm{z} 2=0.0)$ (because this condition is not selected) as shown in Figure 4, where $\mathrm{z} 1$ are CF. The CCF of rule $R_{1111}$ is computed as follows:

For e2: $\mathrm{MB}=0.4+0.0(1-0.4)=0.4, \mathrm{MD}=0.0$;

$\mathrm{MB}+\mathrm{MD}=0.4$

$\mathrm{CCF}=\mathrm{MB}+\mathrm{MD}=0.4$

Evidence in support of rule $R_{1112}$ is: e1: difficulty in locomotion ( $\left.z 1=0.7\right)$. The CCF of rule $R_{1112}$ is 0.7 .

The evidence in support of rule $R_{111}$ are: e1: muscular ( $\left.w=1 / 2, z 1=0.4\right)$ and e2: motor action abnormality $(w=1 / 2, z 2=0.7)$.

Where $w$ is weight and $z 1$ and $z 2$ are CCF at the corresponding node. Therefore, the CCF of $R_{111}$ is computed as follows:

For e1: $\mathrm{z} 1=\mathrm{w}^{*} \mathrm{z} 1=1 / 2^{*} 0.4=0.2$

For e2: $z 2=w^{*} z 2=1 / 2 * 0.7=0.35$

For $\mathrm{e} 2 \mathrm{MB}=0.2+0.35(1-0.2)=0.48, \mathrm{MD}=0.0$;

$\mathrm{CCF}=\mathrm{MB}+\mathrm{MD}=0.48$

Similarly, CCF is computed at other nodes in tree. The complete CCF computation of Schizophrenia is shown in Figure 6. The conclusion should be the disease i.e. Schizophrenia with CCF 0.597. In next section we will apply relative diagnosis and calculate CCF for all diseases.

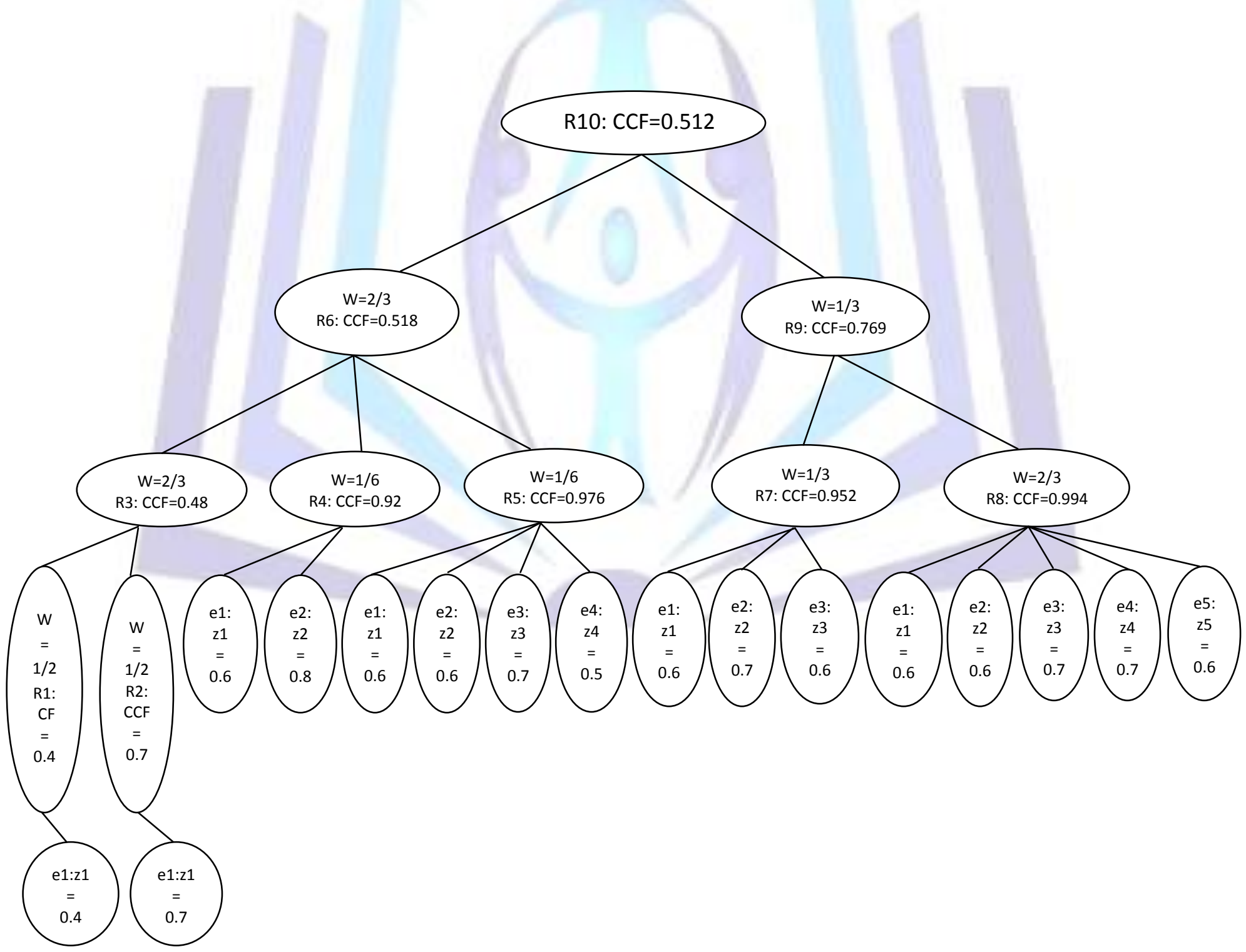

Fig 6: Computation of CCF for Schizophrenia 


\section{COMPUTATION FOR RELATIVE DIAGNOSIS}

The implementation of integrated model of rule base system (RBS) - case base reasoning (CBR) - artificial neural network (ANN) for the diagnosis of neuropsychiatric diseases has been presented. RBS used for generating cases, calculating cumulative confidence factor (CCF). CBR model is used for performing the differential diagnosis (parameters of one disease are considered for the diagnosis of other disease). ANN is use for matching in CBR. The procedure of the CBR reasoning is shown in Figure 7. The portion covered by shaded box is performed by ANN and heuristic methods both. Java language is used for implementing this method. The implementation of the system is done in the following steps: computation of input $(X)$ and output $(Y)$ matrix. Generation of PDV, matching and selection by heuristic and ANN method i.e. finding the row of $X$ matrix that is most similar to PDV and selecting the corresponding row of $Y$ matrix, adaption and computation of CCF of the diseases. Figure 10 shows the entire step in order.

Input case

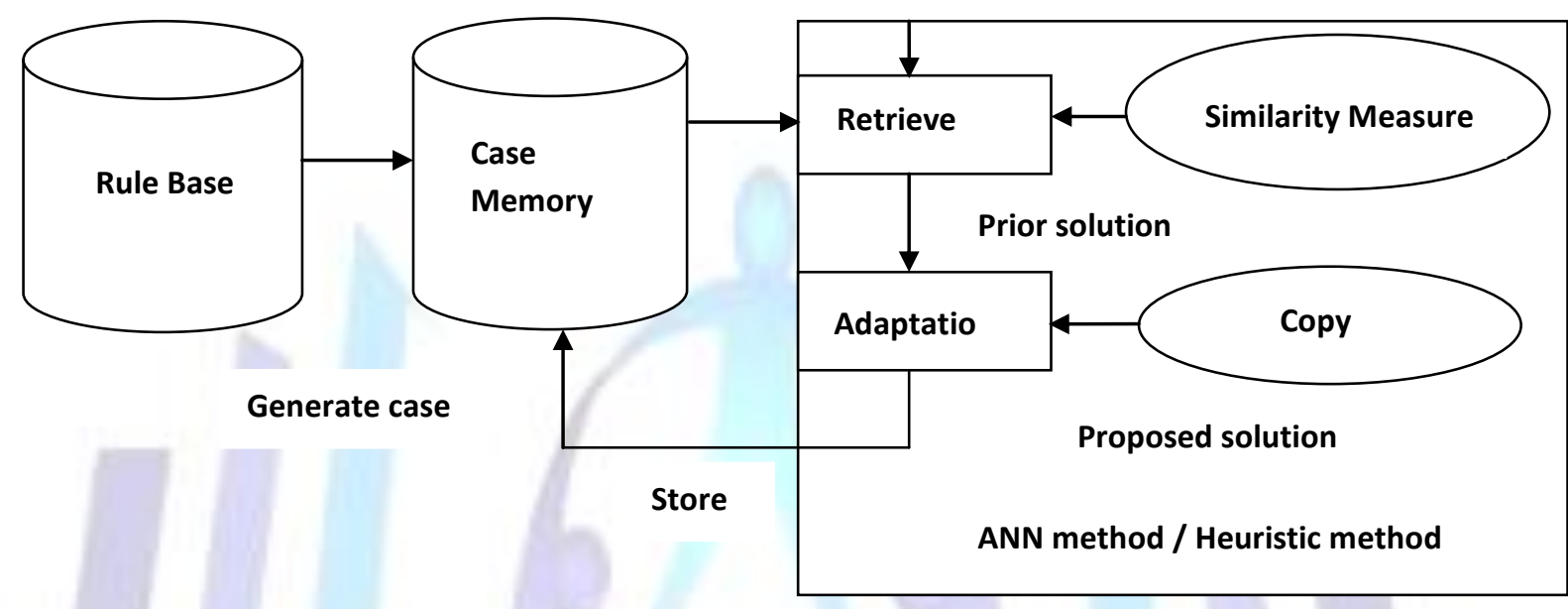

Fig 7: The procedure of the CBR reasoning

\subsection{Case Storage}

The case-base is constructed as a database of the table shape. The table consists of two matrix input $(X)$ matrix (i.e. problem) and output ( $Y$ ) matrix (i.e. solution). Any element $x_{i j}$ of Input matrix $(X)$ is computed as described in Section 5. The pseudo code for the construction of $Y$ matrix from $X$ matrix has been shown in Figure 8. The elements of output matrix $(\mathrm{Y})$ are computed from input matrix $(\mathrm{X})$ as shown below.

Any value of the element of $Y$ matrix is computed as:

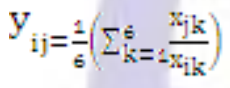

Where $x_{j k}$ is element in $k^{\text {th }}$ column of $j^{\text {th }}$ row of $\mathrm{X}$ matrix, $\mathrm{k}$ is index and $y_{i j}$ is element in $j^{\text {th }}$ column of $i^{\text {th }}$ row of $\mathrm{Y}$ matrix.

The basic purpose to compute $Y$ matrix is to find out relative importance of each of the diseases in comparison to the diseases corresponding to the diagonal element. For example, $\mathrm{y}_{53}$ is computed by dividing the elements of fifth row to the corresponding elements of third row. It means the sign and symptoms of fifth diseases (D5 i.e. Schizophrenia) which corresponds fully to D3, how much relatively it contributes to the diseases D1, D2, D3, D4, and D5, is represented by $\mathrm{y}_{51}$, $\mathrm{y}_{52}, \mathrm{y}_{53}, \mathrm{y}_{54}, \mathrm{y}_{55}$. Each element of $\mathrm{Y}$ matrix is termed as confidence index $(\mathrm{Cl})$.

Each symptom of the particular disease in consideration contributes individually to the sign and symptom of the other diseases i.e. relative (quotient value). For example, symptoms of Mood disorder divided to the symptoms of OCD individually i.e. $F_{41}\left(F_{31} / F_{41}=0.5 / 0.75=0.666\right), F_{42}\left(F_{32} / F_{42}=0.5 / 0.031=16.129\right), F_{43}\left(F_{33} / F_{43}=0.547 / 0.578=0.946\right), F_{44}$ $\left(F_{34} / F_{44}=0.586 / 0.129=4.543\right), F_{45}\left(F_{35} / F_{45}=0.5 / 0.9375=0.533\right)$ and $F_{46}\left(F_{36} / F_{46}=0.766 / 0.937=0.817\right)$. The collective contribution of Mood disorder to Schizophrenia disease is calculated by sum of the relative contribution i.e.

$\mathrm{y}_{43}=\left(\mathrm{F}_{31} / \mathrm{F}_{41}+\mathrm{F}_{32} / \mathrm{F}_{42}+\mathrm{F}_{33} / \mathrm{F}_{43}+\mathrm{F}_{34} / \mathrm{F}_{44}+\mathrm{F}_{35} / \mathrm{F}_{45}+\mathrm{F}_{36} / \mathrm{F}_{46}\right) / 6$

$=(0.666+16.129+0.946+4.543+0.533+0.817) / 6=3.93$

Similarly, other elements of $Y$ matrix are calculated. In case of diagonal elements the value comes to be unity. For example 


$$
\begin{aligned}
\mathrm{y}_{55} & =\left[\left(\sum\left(\frac{\mathrm{x}_{5 k}}{x_{5 k}}\right)\right) / k\right] \\
& =[(0.5 / 0.5+0.25 / 0.25+0.1328 / 0.1328+0.253 / 0.253+0.6875 / 0.6875+0.859 / 0.859) / 6] \\
& =1.0
\end{aligned}
$$

It means sign and symptoms of fifth disease (Schizophrenia) correspond fully to Schizophrenia.

$$
\begin{aligned}
y_{51} & =\left[\left(\sum\left(\frac{x_{1 k}}{x_{5 k}}\right)\right) / k\right] \\
& =[(0.25 / 0.5+0.688 / 0.25+0.2851 / 0.1328+0.563 / 0.253+0.5 / 0.6875+0.578 / 0.859) / 6] \\
& =1.50
\end{aligned}
$$

It means sign and symptoms of fifth disease (Schizophrenia) contributes $(\mid \mathrm{Cl}$ of SI- Cl of ADHD $|=| 1.00-1.50 \mid=0.50)$ $50 \%$ more to the diagnosis of first disease (ADHD) than fifth disease (SI).

Likewise, other elements of $Y$ matrix are interpreted. The $X$ matrix combined with $Y$ matrix formed the case-base as shown in Table 34 below. In Table 4, the values of the F1 to F6 are computed by same steps as described in Section 5.

Table 4. Data of five cases for relative diagnosis (case base)

\begin{tabular}{|l|l|l|l|l|l|l|l|l|l|l|l|}
\hline \multirow{2}{*}{ Diseases } & \multicolumn{9}{|l}{ Input matrix X } & \multicolumn{4}{l|}{ Output matrix Y } \\
\cline { 2 - 13 } & F1 & F2 & F3 & F4 & F5 & F6 & ADHD & Dementia & MD & OCD & SI \\
\hline ADHD & 0.25 & 0.688 & 0.2851 & 0.563 & 0.5 & 0.578 & 1.00 & 1.56 & 1.33 & 1.46 & 0.95 \\
\hline Dementia & 0.75 & 0.625 & 0.742 & 0.283 & 0.6875 & 0.570 & 0.92 & 1.00 & 1.05 & 0.88 & 0.77 \\
\hline MD & 0.5 & 0.5 & 0.547 & 0.586 & 0.5 & 0.766 & 0.85 & 1.37 & 1.00 & 0.98 & 0.77 \\
\hline OCD & 0.75 & 0.031 & 0.578 & 0.129 & 0.9375 & 0.937 & 4.75 & 4.32 & 3.93 & 1.00 & 2.09 \\
\hline SI & 0.5 & 0.25 & 0.1328 & 0.253 & 0.6875 & 0.859 & 1.50 & 2.06 & 1.84 & 1.48 & 1.00 \\
\hline
\end{tabular}

\subsection{Generate PDV (New Case)}

PDV (new case) is generated from user input in the same manner as described in Section 5. New PDV is generated as shown at the bottom most position of the left side of the Figure 9.

$$
\begin{aligned}
& \text { \{Compute output matrix from input matrix\} } \\
& \text { Global parameters: } \\
& \mathrm{X}[1 . . \mathrm{e}][1 . . \mathrm{p}] \text {, matrix stores input data; } \\
& \text { Local variable: } \\
& \mathrm{f:} \mathrm{no} \mathrm{of} \mathrm{parameters;} \mathrm{e:} \mathrm{no} \mathrm{of} \mathrm{diseases;} \\
& \text { Input: } \mathrm{X}[1 . . \mathrm{e}][1 . . \mathrm{f}] \text {, matrix stores input data } \\
& \text { for } i \leftarrow 0 \text { to } f-1 \text { tor } j \leftarrow 0 \text { to } f-1 \\
& \quad \mathrm{y}_{\mathrm{ij}} \leftarrow \frac{1}{5}\left(\sum_{k=0}^{k=5} \frac{\mathrm{x}_{\mathrm{jk}}}{\mathrm{ik}}\right) \\
& \text { end not equal to } \mathrm{j} \text { then } \\
& \text { If } \mathrm{i}==\mathrm{j} \text { then } \\
& \quad \mathrm{y}_{\mathrm{ij}} \leftarrow 0.99 \\
& \text { end if }
\end{aligned}
$$

Fig 8: Pseudo code for computing output matrix $(Y)$ from input matrix $(X)$ 


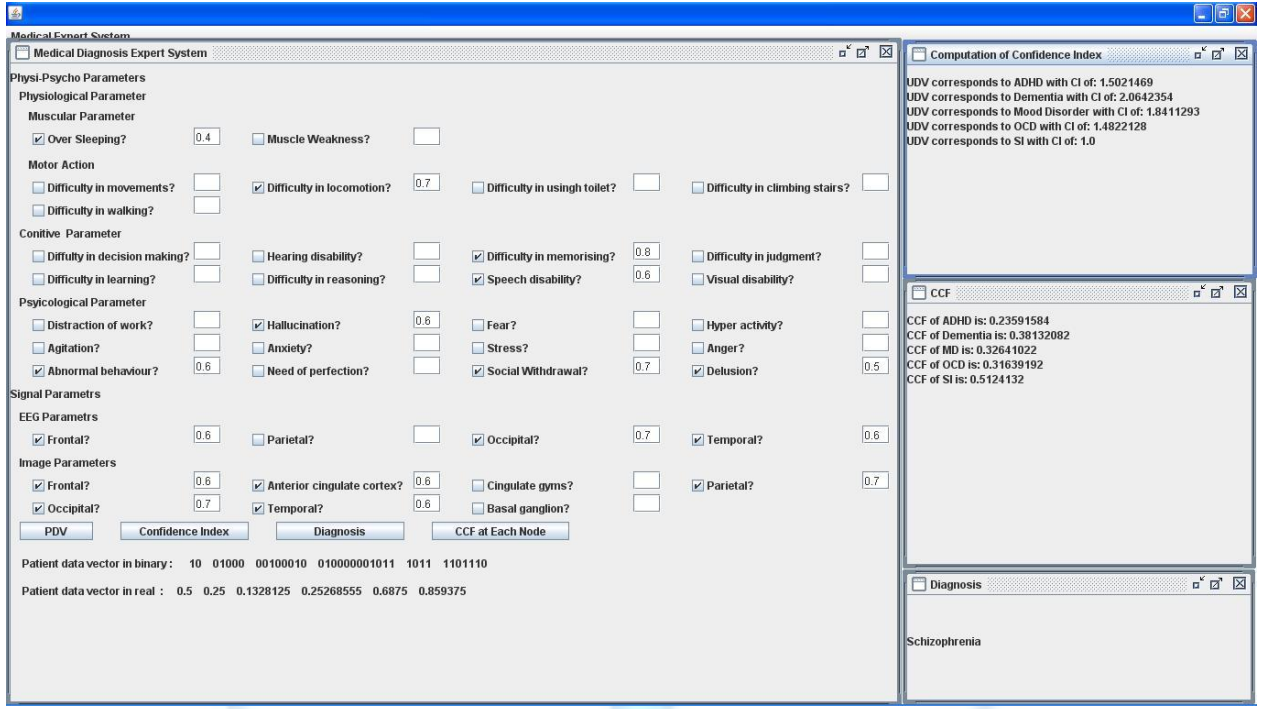

Fig 9: Generation of PDV and computation of CCF

\subsection{Case Retrieval}

In relative diagnosis, case retrieval and matching is performed by two methods: heuristic and ANN method.

Heuristic method: In heuristic method the new case is matched with the cases in case-base using similarity measure described in Section 5. The row, in $Y$ matrix corresponding to minimum $S V_{j}$, where $j=1 . . k$, where $\mathrm{k}$ is the number of cases in the case-base, is selected.

ANN model (matching and learning in CBR): ANN model is used for matching and learning of new cases and situations and then to predict the response or behaviour when the same or the near by cases or situations arise. For developing the neural network, 90 different sets of input-output sets are generated with the slight variation in the input elements of input [X] matrix and corresponding elements in the output [Y] matrix as shown in Table 4.

The ANN model is implemented using MATLAB 6.1 neural network tool. Table 4 is used for training and testing of ANN model. Three layer architectures with back propagation algorithm were deployed with Levenberg Marquardt learning rule. ANN with 6-15-5: input-hidden-output layer was found to be optimum model for matching and selection. Training was terminated at a training error of 10-4 after 88 epochs.

\subsection{Case Adaptation}

Case adaptation is same as described in Section 5.

\subsection{Result and Cumulative Confidence Factor Computation}

Results are shown at different stages of diagnosis process. The first stage is generation of a new PDV as shown at the bottom most position of the left side of the Figure 9. In the next stage the new PDV is matched with the PDVs of stored cases as input matrix $(X)$ as shown in the Table 4. The Cls of the matched case (a row in $X$ ) is selected from a row of the output matrix $(Y)$ in the Table 4. The results of the selected diseases with their respective Cls are shown in the upper position of the right side of Figure 9. Like PDV corresponds to Schizophrenia with $\mathrm{Cl} 1.0$ and so on.

The result is illustrated as follows: Assume that a PDV1 is presented (new case1). The symptoms of the PDV1 are shown in Figure 9.

The signs and symptoms of the patient shown in the PDV correspond fully $(\mathrm{Cl}=1.0)$ to the disease Schizophrenia as shown in Figure 9. The same PDV corresponds to different values of $\mathrm{Cl}$ in the diagnosis of other diseases. For example, the same PDV correspond to ADHD with $(\mid \mathrm{Cl}$ Schizophrenia of $-\mathrm{Cl}$ of $\mathrm{ADHD}|=| 1.0-1.502 \mid=0.502) 50.2 \% \mathrm{higher} \mathrm{Cl}$ then Schizophrenia, Dementia with $(\mid \mathrm{Cl}$ Schizophrenia of $-\mathrm{Cl}$ of Dementia $|=| 1.0-2.064 \mid=1.06) 106 \%$ higher $\mathrm{Cl}$ then Schizophrenia, Mood Disorder with $(\mid \mathrm{Cl}$ Schizophrenia of $-\mathrm{Cl}$ of $\mathrm{MD}|=| 1.0-1.84 \mid=0.84) 84 \%$ higher $\mathrm{Cl}$ then Schizophrenia, OCD with $(\mid C I$ Schizophrenia of $-\mathrm{CI}$ of $\mathrm{OCD}|=| 1.0-1.48 \mid=0.48) 48 \%$ higher $\mathrm{Cl}$ then Schizophrenia. The above results are obtained by heuristic method. 
When ANN model is applied for matching and learning the output of the PDV1 $\{0.5,0.25,0.1328125,0.25268555$, $0.6875,0.859375\}$ is $\{1.59,2.18,1.82,1.49,0.96\}$ as shown in Table 5 . The output is near to the first row in $Y$ matrix which means the PDV1 is matched to Schizophrenia by ANN method also.

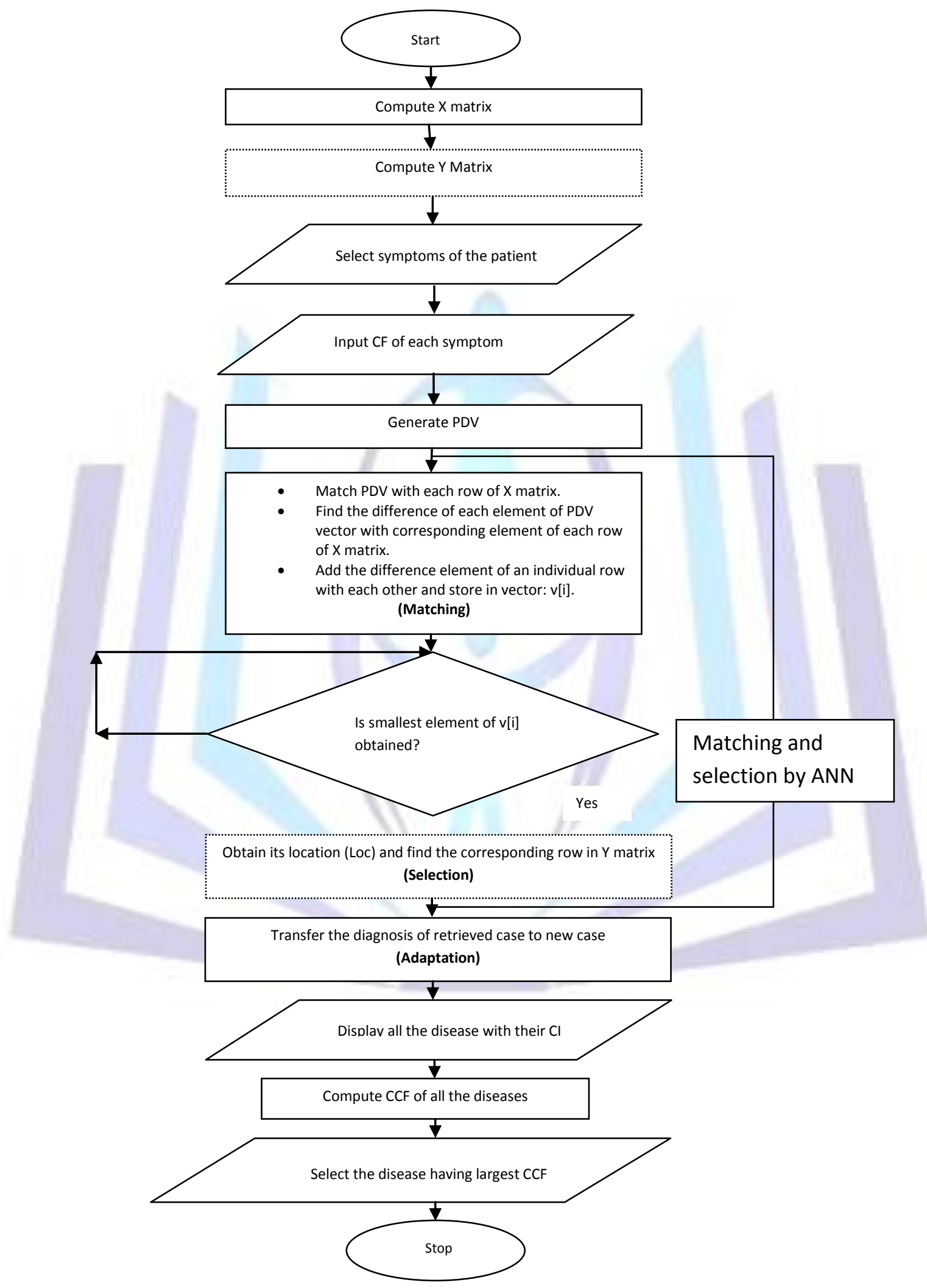

Fig 10: Flowchart for implementation of relative diagnosis 
The result obtained by both methods are nearly same but the matching is done by ANN model in only one step whereas the matching and learning in heuristic is done in three steps as shown in pseudo code in Figure 15 . Therefore, The ANN model is faster then heuristic method.

Similarly, we tested other two unknown PDVs: PDV2 and PDV3 by ANN and heuristic methods and the difference between the results of the two methods are shown in Table 5. It is clear that difference is insignificant as shown by error in the output matrix of Table 5.

Table 5. PDV and its predicted output by ANN

\begin{tabular}{|c|c|c|c|c|c|c|c|c|c|c|c|c|}
\hline \multirow[t]{2}{*}{ PDV } & \multicolumn{6}{|c|}{ Input } & \multirow[t]{2}{*}{ Approach/error } & \multicolumn{5}{|c|}{ Output } \\
\hline & F1 & F2 & F3 & F4 & F5 & F6 & & 01 & 02 & $\mathbf{0 3}$ & 04 & 05 \\
\hline \multirow[t]{3}{*}{ PDV1 } & \multirow[t]{3}{*}{0.5} & \multirow[t]{3}{*}{0.25} & \multirow[t]{3}{*}{0.1328} & \multirow[t]{3}{*}{0.253} & \multirow[t]{3}{*}{0.6875} & \multirow[t]{3}{*}{0.859} & ANN (CIANN) & 1.59 & 2.18 & 1.82 & 1.49 & 0.96 \\
\hline & & & & & & & Heur $(\mathrm{CIH})$ & 1.50 & 2.06 & 1.84 & 1.48 & 1.00 \\
\hline & & & & & & & Error & 0.09 & 0.12 & 0.02 & 0.01 & 0.04 \\
\hline \multirow[t]{3}{*}{ PDV2 } & \multirow[t]{3}{*}{0.75} & \multirow[t]{3}{*}{0.031} & \multirow[t]{3}{*}{0.742} & \multirow[t]{3}{*}{0.129} & \multirow[t]{3}{*}{0.9375} & \multirow[t]{3}{*}{0.937} & ANN (CIANN) & 4.68 & 4.39 & 3.88 & 0.95 & 2.18 \\
\hline & & & & & & & Heur $(\mathrm{CIH})$ & 4.75 & 4.32 & 3.93 & 1.00 & 2.09 \\
\hline & & & & & & & Error & 0.07 & 0.07 & 0.05 & 0.05 & 0.09 \\
\hline \multirow[t]{3}{*}{ PDV3 } & \multirow[t]{3}{*}{0.75} & \multirow[t]{3}{*}{0.625} & \multirow[t]{3}{*}{0.742} & \multirow[t]{3}{*}{0.283} & \multirow[t]{3}{*}{0.5} & \multirow[t]{3}{*}{0.578} & ANN (CIANN) & 0.93 & 0.99 & 1.13 & 0.91 & 0.63 \\
\hline & & & & & & & Heur $(\mathrm{CIH})$ & 0.92 & 1.00 & 1.05 & 0.88 & 0.77 \\
\hline & & & & & & & Error & 0.01 & 0.01 & 0.08 & 0.07 & 0.14 \\
\hline
\end{tabular}

The CCFs of all the diseases are shown in middle of right side of the Figure 9 and also shown in Table 6.

Table 6. CCF computed for all diseases

\begin{tabular}{|l|l|}
\hline Disease & CCF \\
\hline ADHD & 0.235 \\
\hline Dementia & 0.381 \\
\hline Mood Disorder & 0.326 \\
\hline OCD & 0.316 \\
\hline SI & 0.512 \\
\hline
\end{tabular}

The conclusion should be the disease, which has the highest CCF. Thus, the diagnosis is Schizophrenia with CCF 0.512. The CCFs shown in Figure 9 and Table 6 are computed through the program. The different steps for computation of CCFs in case of all diseases are also shown manually to illustrate it with respect to Figure 6 and Figure 11 to 14. CCF is computed by same method described in Section 3. The CCF at each node at the different levels of the hierarchy is shown in Figure 6 and Figure 11 to 14.

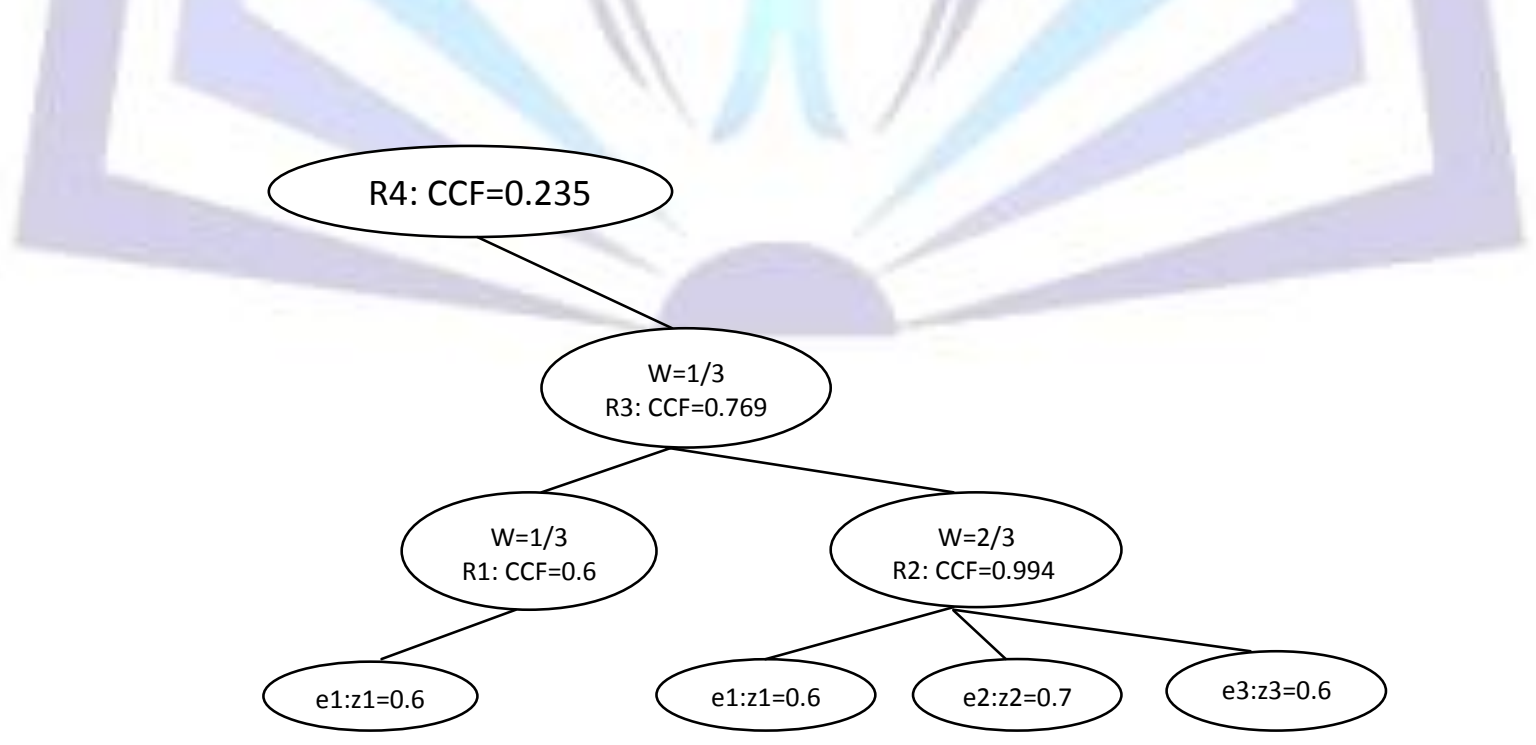

Fig 11: Computation of CCF for ADHD 


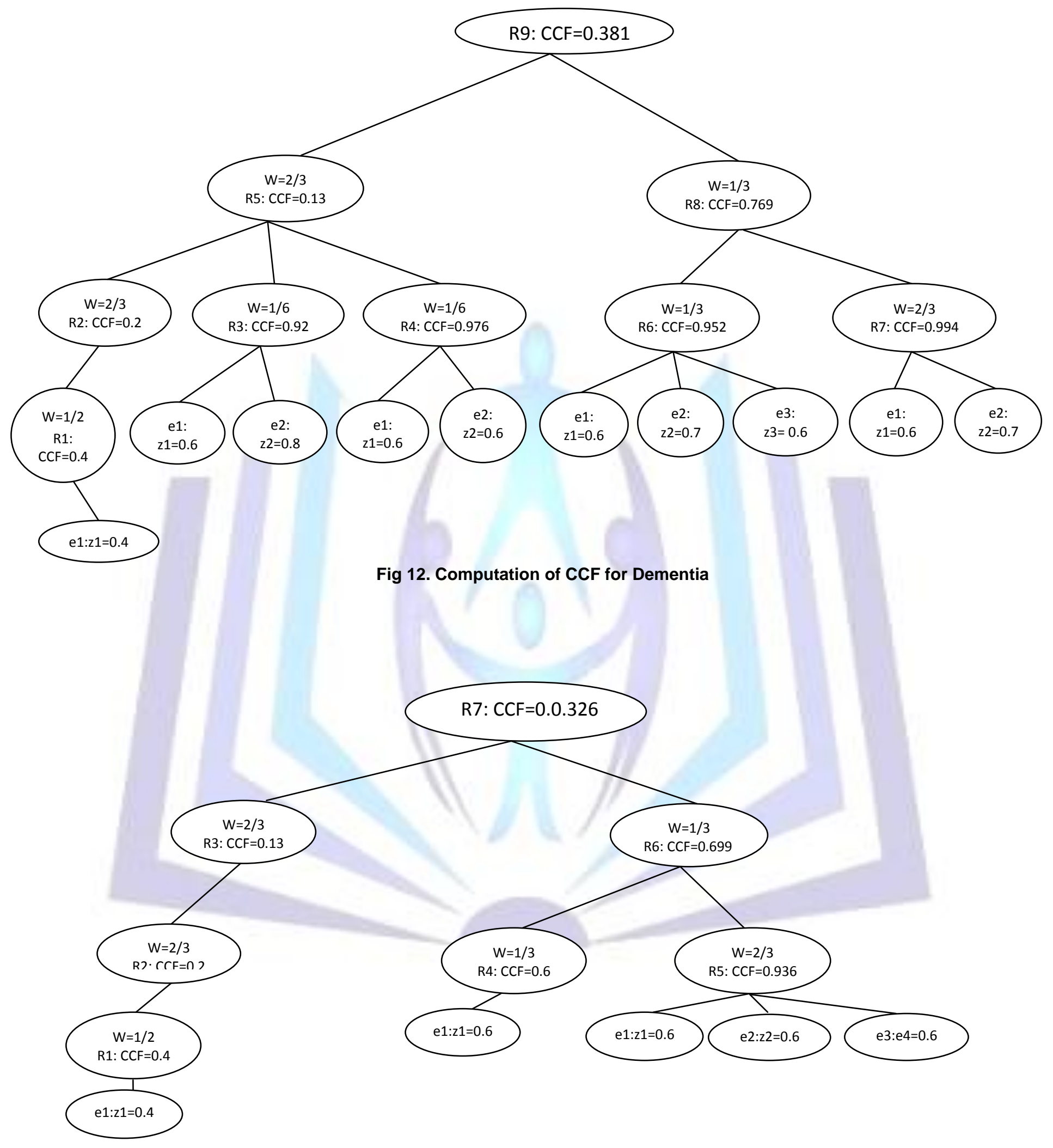

Fig 13: Computation of CCF for Mood Disorder 


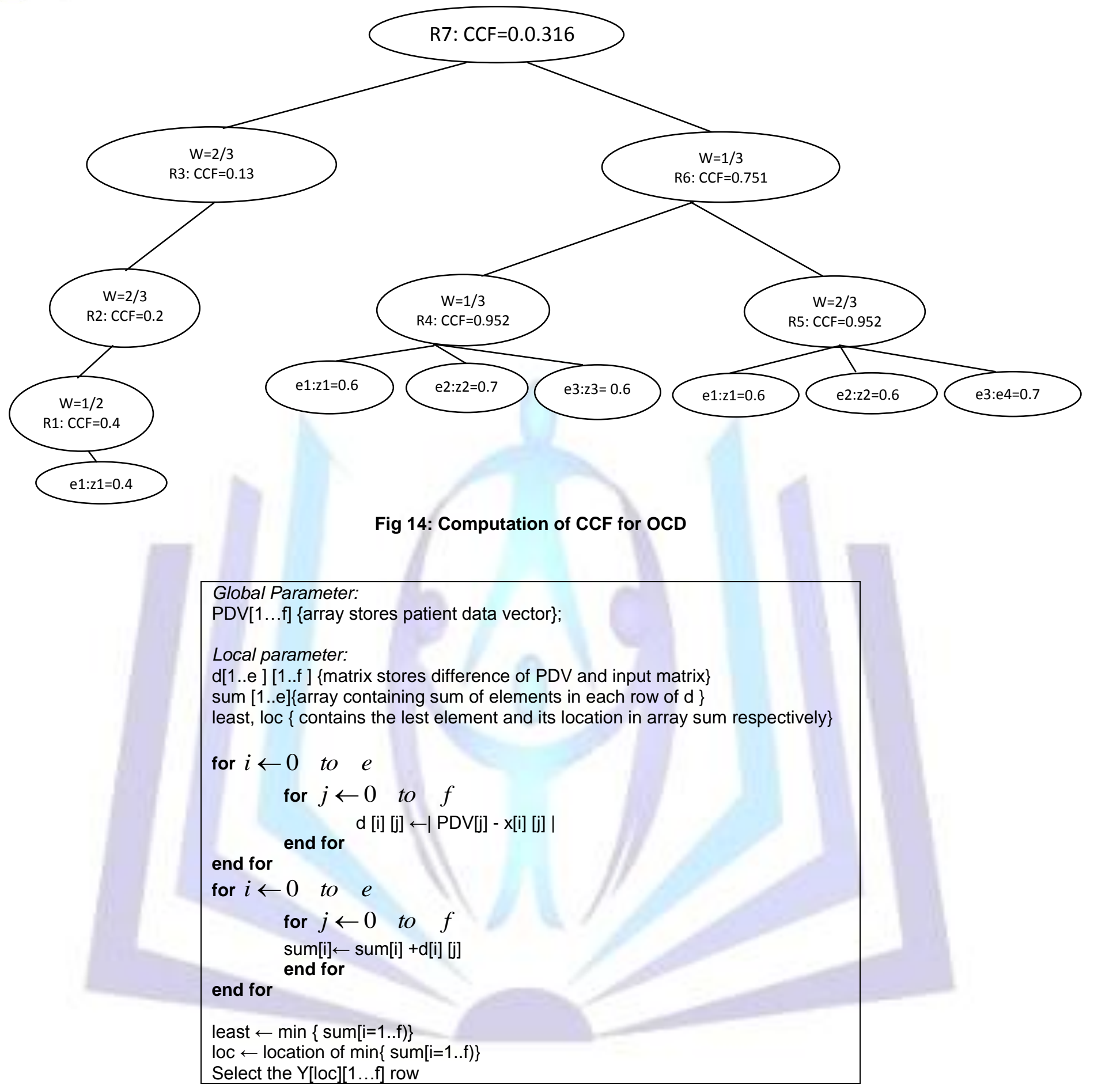

Fig 15: Pseudo code for matching and selection in CBR

\section{CONCLUSION}

The methods RBR and CBR have been combined for the absolute diagnosis of neuropsychiatric disease in a heuristic manner. Absolute diagnosis means that the sign and symptoms of one disease may correspond to only one disease. The methods RBR, CBR and ANN have been combined for the relative diagnosis of neuropsychiatric diseases in a heuristic manner. The RBR approach shows a hierarchical correlation of sign and symptom with the diseases. The hierarchical tree is used in computing the cumulative confidence factor and to develop the case-base. The sign and symptoms of one disease which confirms it with unity is correlated by its contribution to other diseases by computation of the elements of $Y$ matrix. The difference of the element of $Y$ matrix (diagonal element) with unity $\mathrm{Cl}$ to other elements of the $\mathrm{Y}$ matrix (non diagonal elements) in the same row yields the relative contribution of sign and symptoms of one disease (with unity $\mathrm{Cl}$ ) to other disease (with non unity $\mathrm{Cl}$ ). This is our significant contribution in this work. 
A combined structure, depicting the sign and symptom displayed in the physical, psychological and cognitive, EEG and neuroimagin parameters have rarely been developed for diagnosis of neuropsychiatric diseases and also an integrated computing model such as: RBS, CBR and ANN have also rarely been used for diagnosis of neuropsychiatric diseases. We have made an effort to develop a combined methodology and use it effectively and efficiently. Effectively in the sense, that the combined method performs the job of computation collectively which an individual method may not be able to perform. Efficiently in the sense, that this methodology covers the data in the form of sign and symptoms and knowledge in the form of rules and reasoning procedure of CBR augmented with ANN method.

The sign and symptoms of neuropsychiatric diseases have been taken from literature, consultation with physician and researchers in the domain area. The sign, symptoms and confidence factor may be altered or become unanimously in consultation with more number of physicians and experimentations in clinical settings. Experimentation in clinical setting would confirm the effectiveness of our method which has also a scope for further work. However, we claim the effectiveness of our computing model rather then the experimentation results of the model.

According to our study, we make following observation:

a) When we apply the CBR, the new case1 is similar to the Schizophrenia with $\mathrm{Cl}$ value 1.00 . When we apply the method RBR for calculating the CCF for all the diseases, RBR calculate highest CCF value for Schizophrenia. The combined result i.e. when we apply both the methods gives the conclusion "Schizophrenia".

b) Applying the combining method, one can incorporate the documented experience of the case-base and the expert experience of rule-base. Thus the combining conclusion should increase the accuracy over any single method applied alone.

c) Addition to diagnosis of disease the CBR approach predicts the order of confidence of disease i.e. how much the given symptoms and parameter contributes to the other diseases in relative diagnosis. The result from ANN model and heuristic model are nearly same but the matching and selection by ANN model is fast.

\section{REFERENCES}

[1] Prentzas, Jim, and loannis Hatzilygeroudis. "Categorizing approaches combining rule-based and case-based reasoning." Expert Systems 24.2 (2007): 97-122.

[2] Handelman, D. A., Stephen H. Lane, and Jack J. Gelfand. "Integrating neural networks and knowledge-based systems for intelligent robotic control." Control Systems Magazine, IEEE 10.3 (1990): 77-87.

[3] Dragulescu, Doina, and Adriana Albu. "Expert system for medical predictions." Applied Computational Intelligence and Informatics, 2007. SACl'07. 4th International Symposium on. IEEE, 2007.

[4] Marling, Cindy, and Peter Whitehouse. "Case-based reasoning in the care of Alzheimer's disease patients." Casebased reasoning research and development. Springer Berlin Heidelberg, 2001. 702-715.

[5] Park, Hae-Jeong, et al. "Automated sleep stage scoring using hybrid rule-and case-based reasoning." Computers and Biomedical Research 33.5 (2000): 330-349.

[6] Yudofsky, Stuart C., and Robert E. Hales. "The reemergence of neuropsychiatry: definition and direction." J Neuropsychiatry Clin Neurosci. 1989 Winter; 1(1):1-6.

[7] Yudofsky, Stuart C., and Robert E. Hales. "Neuropsychiatry and the future of psychiatry and neurology." American Journal of Psychiatry 159.8 (2002): 1261-1264.

[8] Yudofsky, Stuart C., and H. Florence Kim, eds. Neuropsychiatric Assessment. Vol. 22. American Psychiatric Pub, 2008.

[9] Cummings, Jeffrey L., and Michael R. Trimble. Concise guide to neuropsychiatry and behavioral neurology. American Psychiatric Pub, 2008.

[10] Lajara-Nanson WA, Schiffer RB. "Neuropsychiatry". In: Baker's clinical neurology, cd-rom. Philadelphia: Lippincott Williams \& Wilkins, 2001.

[11] DeJong R, "The Neurologic Examination." 4th Ed. New York: Harper and Row, 1979.

[12] Strub RI, Black FW. "The Mental Status Examination in Neurology," 3rd ed. Philadelphia, Pa: FA Davis; 1993.

[13] Trzepacz PT, Baker RW. "The Psychiatric Mental Status Examination." New York: Oxford University Press, 1993.

[14] Weintraub, S., \& Mesulam, M. M. "Mental state assessment of young and elderly adults in behavioral neurology." Principles of behavioral neurology, 26 (1985), 71-123.

[15] Schiffer, Randolph B., Stephen M. Rao, and Barry S. Fogel, eds. Neuropsychiatry. Wolters Kluwer Health, 2003.

[16] Sanei, Saeid, and Jonathon A. Chambers. "EEG signal processing." Wiley. com, 2008. 
[17] Shenton, Martha E., and Bruce I. Turetsky, eds. "Understanding neuropsychiatric disorders: Insights from Neuroimaging." Cambridge University Press, 2010.

[18] American Psychiatric Association. "Diagnostic and statistical manual of mental disorders" (5th ed.). Arlington, VA: American Psychiatric Publishing, (2013).

[19] World Health Organization, "The ICD-10 classification of mental and behavioural disorders." Clinical descriptions and diagnostic guidelines. WHO, Geneva, (1992).

[20] Miyoshi, Koho, and Yasushi Morimura. "Clinical Manifestations of Neuropsychiatric Disorders." Neuropsychiatric Disorders. Springer Japan, 2010. 1-14.

[21] Meddahi, J. Karim, and Ben H. Jansen. "Knowledge acquisition for multi-channel electroencephalogram interpretation." Artificial Intelligence in Medicine 4.5 (1992): 315-328.

[22] García-Pérez E, Violante A, Cervantes-Pérez F. "Using neural networks for differential diagnosis of Alzheimer disease and vascular dementia." Expert Systems with Applications (vol 14). Elsevier, 1998: 219-225.

[23] Zou, Y., Shen, Y., Shu, L., Wang, Y., Feng, F., Xu, K., \& Liu, W. "Artificial neural network to assist psychiatric diagnosis." The British Journal of Psychiatry, 169(1), 64-67., 1996.

[24] Y.-J. Li and F.-Y. Fan, "Classification of Schizophrenia and depressionby EEG with ANNs," in Proceedings Int. Conf. of the IEEE Eng. in Medicine and Biology Society,pp. 2679-2682, Sept. 2005.

[25] Yana, Kazuo, et al. "A neural net screening of psychiatric patients." Engineering in Medicine and Biology Society, 1994. Engineering Advances: New Opportunities for Biomedical Engineers. Proceedings of the 16th Annual International Conference of the IEEE. IEEE, 1994.

[26] Vollala, Satyanarayana, and Karnakar Gulla. "Automatic Detection of EPILEPSY EEG Using Neural Networks."

[27] Lowell WE, Davis GE. "Predicting length of stay for psychiatric diagnosis-related groups using neural networks." J Am Med Informat Assoc. 1: 459-466, 1994.

[28] Reategui, E., Campbell, J. A., \& Borghetti, S. "Using a neural network to learn general knowledge in a case-based system." In Case-Based Reasoning Research and Development (pp. 528-537). Springer Berlin Heidelberg, 1995.

[29] Haraldsson, H., Edenbrandt, L., \& Ohlsson, M. "Detecting acute myocardial infarction in the 12-lead ECG using Hermite expansions and neural networks." Artificial Intelligence in Medicine, 32(2), 127-136, (2004).

[30] Shortliffe, Edward H., and Bruce G. Buchanan. "A model of inexact reasoning in medicine." Mathematical biosciences 23.3(1975):351-379. 